\title{
Dual-Functional Plasmonic Photothermal Biosensors for Highly Accurate Severe Acute Respiratory Syndrome Coronavirus 2 Detection
}

Guangyu Qiu, Zhibo Gai, Yile Tao, Jean Schmitt, Gerd A. Kullak-Ublick, and Jing Wang*

Cite This: ACS Nano 2020, 14, 5268-5277

Read Online

ACCESS 1

Џll Metrics \& More

回国 Article Recommendations

Supporting Information

ABSTRACT: The ongoing outbreak of the novel coronavirus disease (COVID-19) has spread globally and poses a threat to public health in more than 200 countries. Reliable laboratory diagnosis of the disease has been one of the foremost priorities for promoting public health interventions. The routinely used reverse transcription polymerase chain reaction (RT-PCR) is currently the reference method for COVID-19 diagnosis. However, it also reported a number of false-positive or -negative cases, especially in the early stages of the novel virus outbreak. In this work, a dual-functional plasmonic biosensor combining the plasmonic photothermal (PPT) effect and localized surface plasmon resonance (LSPR) sensing

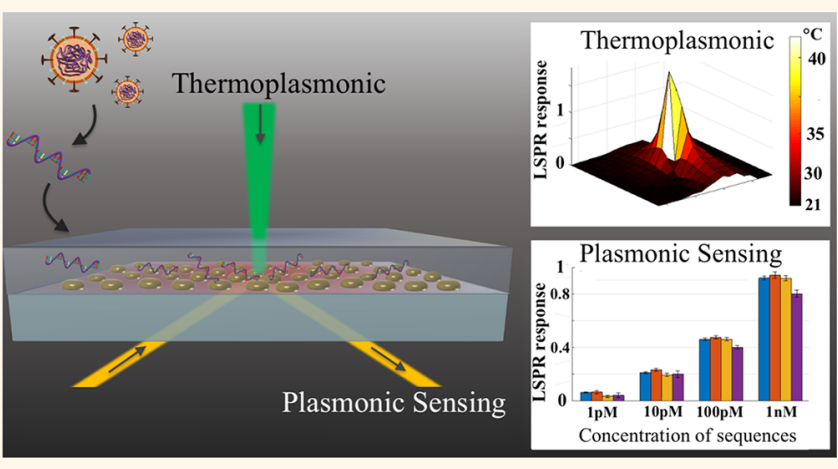
transduction provides an alternative and promising solution for the clinical COVID-19 diagnosis. The two-dimensional gold nanoislands (AuNIs) functionalized with complementary DNA receptors can perform a sensitive detection of the selected sequences from severe acute respiratory syndrome coronavirus 2 (SARS-CoV-2) through nucleic acid hybridization. For better sensing performance, the thermoplasmonic heat is generated on the same AuNIs chip when illuminated at their plasmonic resonance frequency. The localized PPT heat is capable to elevate the in situ hybridization temperature and facilitate the accurate discrimination of two similar gene sequences. Our dual-functional LSPR biosensor exhibits a high sensitivity toward the selected SARS-CoV-2 sequences with a lower detection limit down to the concentration of $0.22 \mathrm{pM}$ and allows precise detection of the specific target in a multigene mixture. This study gains insight into the thermoplasmonic enhancement and its applicability in the nucleic acid tests and viral disease diagnosis.

KEYWORDS: plasmonic photothermal effect, severe acute respiratory syndrome coronavirus 2, coronavirus disease, LSPR, biosensors, nuclei acids, RNA virus

\section{A} $t$ the end of 2019, the first case of pneumonia of unknown origin was detected in Wuhan, China. ${ }^{1}$ Highthroughput sequencing revealed that this was a new severe acute respiratory syndrome $\beta$-coronavirus (SARS-CoV2 ) and a novel coronavirus disease (COVID-19). ${ }^{2}$ Through 1 April 2020, the rapid spread of COVID-19 has impacted more than 200 countries with more than 900000 laboratoryconfirmed cases and 45000 deaths (with high numbers in China, United States, Spain, and Italy). ${ }^{3,4}$ COVID-19 is the third large-scale pandemic caused by coronavirus in the last two decades after severe acute respiratory syndrome (SARS) in 2003 and Middle East Respiratory Syndrome (MERS) in 2012. ${ }^{5,6}$ These two coronaviruses have caused about 10000 cumulative cases, with mortality rates of $10 \%$ for SARS-CoV and 37\% for MERS-CoV. Regarding the SARS-CoV-2, the laboratory-confirmed COVID-19 cases have already been more than 90 times higher than the total confirmed cases of SARS and MERS. ${ }^{7}$ There is no doubt that fast and accurate identification of a novel virus can greatly contribute to the control of an emerging pandemic.

Received: March 21, 2020

Accepted: April 8, 2020

Published: April 13, 2020

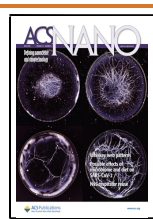


(a)

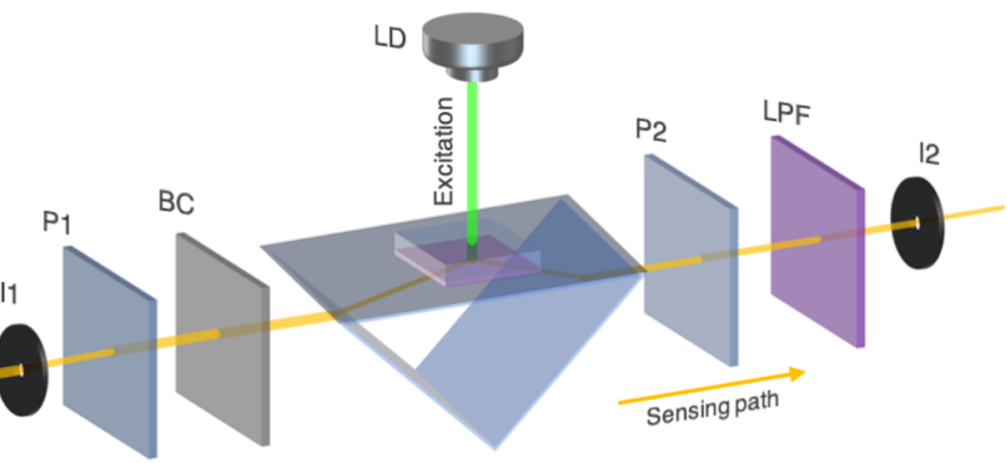

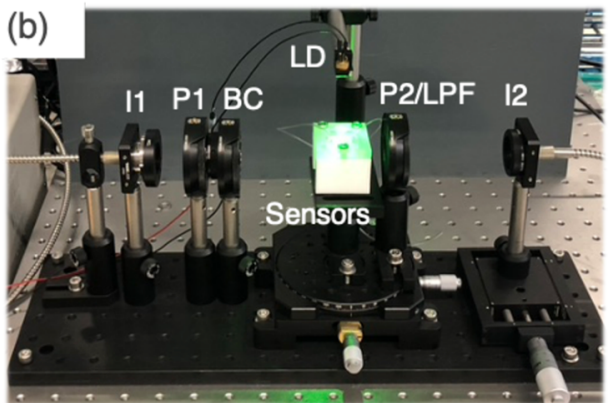

(c)

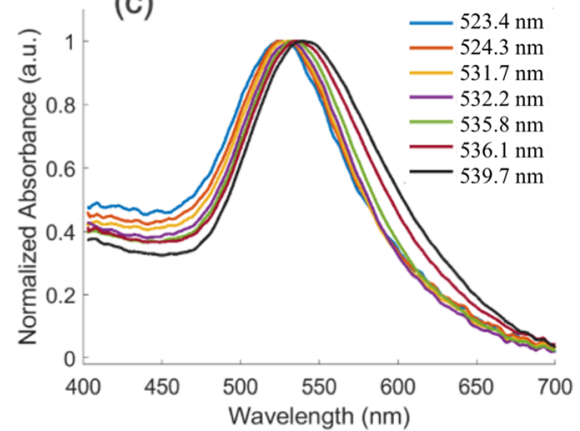

(d)

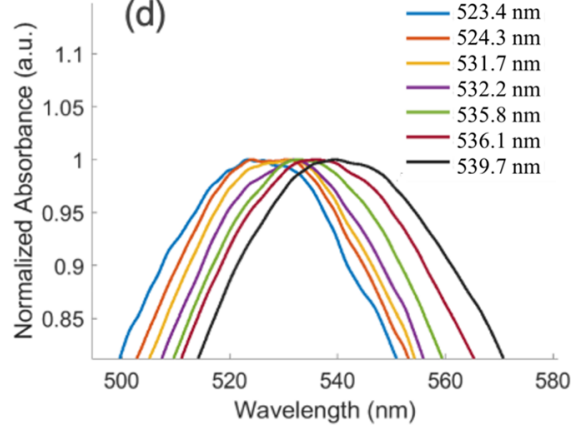

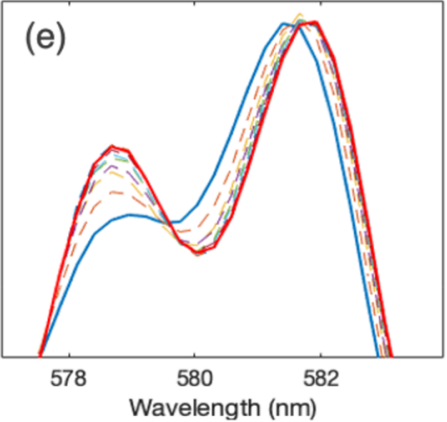

Figure 1. Experimental setup and system optimization. (a) Schematic and (b) experimental setup of the dual-functional PPT enhanced LSPR biosensing system. In the LSPR sensing path, the collimated wide spectrum beam passed through the aperture-iris (I1/I2), the linear polarizers (P1/P2), the birefringent crystal (BC), and totally reflected at the interface of AuNI-dielectric for LSPR detection. In the excitation unit, a laser diode (LD) was used to generate the PPT effect on AuNIs in the normal incident angle. (c, d) Normalized absorbances of the AuNI sensor chips showing a fine-tune peak absorption from 523.4 to $539.7 \mathrm{~nm}( \pm 0.2 \mathrm{~nm})$. (e) Plasmonic resonance wavelength at about $580 \mathrm{~nm}$ under the ATR (attenuated total reflection) configuration for LSPR sensing transduction.

Reliable laboratory diagnosis has been one of the foremost priorities for promoting epidemic prevention and control. In acute respiratory infection, the molecular method reverse transcription polymerase chain reaction (RT-PCR) is routinely used to detect causative viruses using samples from respiratory secretions. $^{8}$ According to the latest version of "WHO interim guidance for laboratory testing for COVID-19 in humans", several molecular assays that detect the COVID-19 have been developed. ${ }^{9}$ The gene targets for RT-PCR molecular assays selected by different countries are genetically similar, including the RNA-dependent RNA polymerase (RdRp) sequence and the open reading frame $1 \mathrm{ab}$ (ORF1ab) sequence. Generally, RT-PCR is currently the most sensitive method of viral RNA detection by rapidly making many copies of a specific sequence. The sensitivity of a recent SARS-CoV-2 study has reached 3.7 RNA copies on detecting the RdRp sequence. ${ }^{8}$ However, RT-PCR can also fail for various reasons, such as its amplification of spurious nucleic acid contaminations. The RTPCR assays for SARS-CoV-2 detection have reported a number of false-negative results on confirmed infection cases. ${ }^{10}$ In clinical diagnosis, a single negative PCR result does not rule out COVID-19 infection as the reported positive rate was only $30-50 \%$ for laboratory-confirmed COVID-19 cases at the early stage of the outbreak, ${ }^{11}$ particularly if the sample is from an upper respiratory tract (URT) specimen. A recent study of 167 COVID-19 infection patients showed that five $(3 \%)$ patients had positive chest computed tomography (CT) findings but false-negative results from the RT-PCR testing. These five patients were eventually confirmed with COVID-19 infection by repeated swab tests. ${ }^{10}$ In addition, the current RT-PCR-based detection methods demand high manpower and long processing time, which may not be able to provide the capacity to test all the suspected cases during full-scale outbreaks. Other approaches such as CT scan and culture methods are apparently not suitable for fast-response detection and real-time analysis. ${ }^{12}$ Therefore, it is advantageous to thoroughly investigate suspected patients by another reliable diagnosis system.

Biosensors are ideal for providing an alternative and reliable solution to clinical diagnosis, real-time detection, and continuous monitoring. ${ }^{13,14}$ Among the different biosensing techniques, localized surface plasmon resonance (LSPR) biosensing systems are applicable to different classes of analytes of clinical interests. ${ }^{15}$ LSPR is a strong photon-driven coherent oscillation of the surface conduction electrons, which can be modulated when coupling occurs at the surface of the plasmonic materials. ${ }^{16}$ Owing to the enhanced plasmonic field in the vicinity of the nanostructures, LSPR sensing systems demonstrate high sensitivity to local variation, including the refractive index change and molecular binding. ${ }^{17}$ Thus, LSPR is an ideal candidate for real-time and label-free detection of micro- and nanoscale analytes. ${ }^{18,19}$ A latest research has utilized SPR to test the biophysical properties of SARS-CoV-2 spike protein and found that the SARS-CoV-2 spike glycoprotein bound angiotensin-converting enzyme 2 (ACE2) with much higher affinity than SARS-CoV spike protein. ${ }^{20}$ In addition, several SARS-CoV receptor-binding domains (RBDs)-specific monoclinal antibodies were also tested in this study and demonstrated that these antibodies did not have appreciable binding to the spike protein of SARS$\mathrm{CoV}-2$. The key property of nucleic acids that renders them so useful for clinical diagnosis, therapy and bionanotechnology is 
(a)

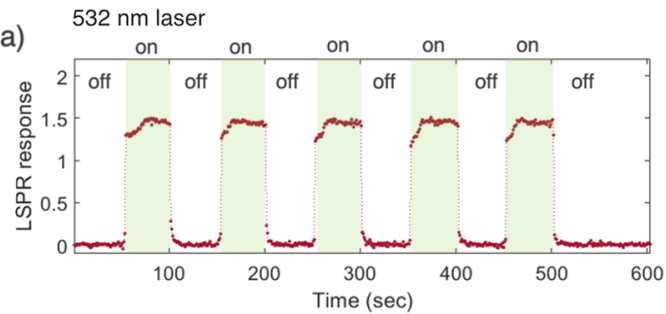

(d)

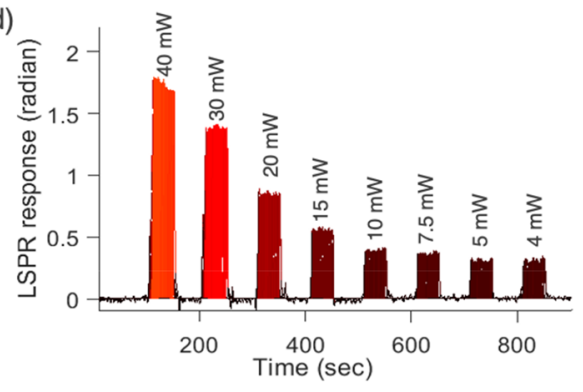

(b)

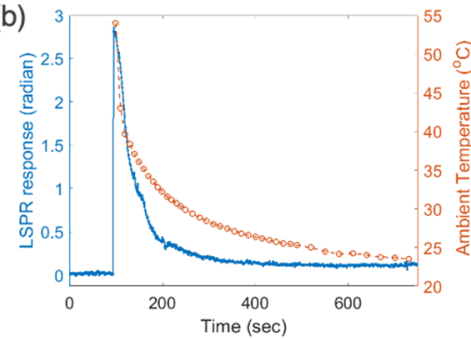

(e)

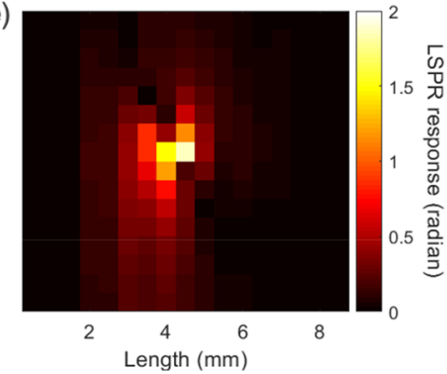

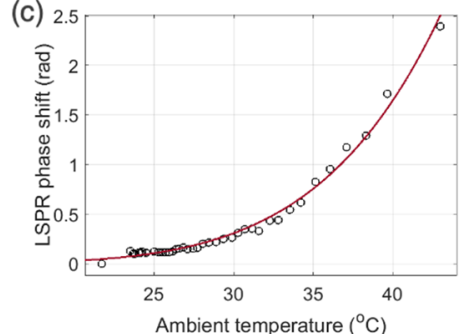

(f)

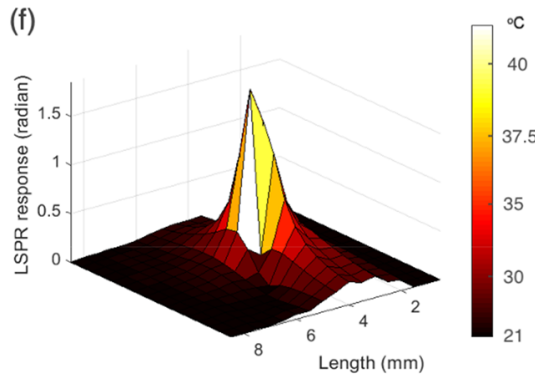

Figure 2. In situ characterization of local PPT heating on AuNIs. (a) Periodic laser excitation and the PPT-induced plasmonic phase response. (b) Temperature variations and real-time LSPR responses. (c) Calibration curve illustrating the relationship between the temperature and LSPR phase response. (d) Real-time LSPR responses caused by the laser-induced PPT effect under different laser powers. (e) Scanned local LSPR responses around the PPT heat source on AuNIs. (d) Mapping the temperature distribution around the PPT heat source.

the predictable and specific hybridization of complementary bases. ${ }^{21}$ Thus, the LSPR technique for genetic testing and nucleic acid detection in clinical practices could be an interesting alternative for SARS-CoV-2 detection and COVID-19 diagnosis.

The novel SARS-CoV-2 virus is a positive sense, singlestranded RNA virus. The DNA-RNA hybridization has been widely used in RT-PCR as well as various biomedical sensors. The criteria for hybridization are based on nucleic acid strand melting. ${ }^{22,23}$ Two complementary strands can specifically hybridize with each other when the temperature is slightly lower than their melting temperature, while a single mismatch can cause the melting temperature to decrease significantly. ${ }^{24}$ It is worth noting that the plasmonic nanoparticles normally exhibit large optical cross sections and the absorbed light can be nonradiatively relaxed resulting in a significant heating energy. ${ }^{25,26}$ The converted plasmonic photothermal (PPT) heat energy, also known as the thermoplasmonic effect is highly localized near the nanoparticles, which can be used as a stable in situ heat source for controllable and uniform thermal processing. $^{26-29}$ In this work, we developed a dual-functional LSPR biosensor through combining the photothermal effect and plasmonic sensing transduction for SARS-CoV-2 viral nucleic acid detection. The plasmonic chip with the twodimensional distribution of nanoabsorbers (AuNIs) is capable to generate the local PPT heat and transduce the in situ hybridization for highly sensitive and accurate SARS-CoV-2 detection.

\section{RESULTS AND DISCUSSION}

The dual-functional plasmonic performances were systematically studied in the aspects of LSPR sensing transduction and PPT heating. The common-path differential phase-sensitive LSPR system, as shown in Figure 1a, was adopted to measure the local refractive index changes or the binding events. In the LSPR sensing transduction unit, the sensing beam was generated by a wide spectrum LED source and operated in the ATR (attenuated total reflection) mode at the interface between the glass substrate and liquid environment. When reaching the two-dimensional AuNI sensing layer, the measured optical power of the beam was found to be 32.58 $\mu \mathrm{W}$. The local plasmonic responses were retrieved from the ATR spectral interferograms by using the windowed Fourier transform phase extraction method, as described elsewhere. ${ }^{30}$ This phase response, reported in radian units, is more prominent than the conventional spectral and angular responses. Therefore, it has been utilized for improving the sensitivity of plasmonic sensors. ${ }^{31}$ In order to generate a stable and intense thermoplasmonic field, an excitation laser with 532 $\mathrm{nm}$ peak wavelength and $40 \mathrm{~mW}$ maximum optical power was applied onto the AuNI chip in the normal incident angle (Figure 1b). In addition, optimizing the AuNI chip so that its peak absorbance wavelength was exactly at $532 \mathrm{~nm}$ can significantly improve the conversion efficiency of thermoplasmonic. By adjusting the $\mathrm{Au}$ nanofilm thickness before dewetting, the absorption peak (under normal incident angle) can be accurately controlled within a wavelength range from 523.4 to $539.7 \mathrm{~nm}$ as shown in Figure 1c,d and Figure S1. In this work, the AuNIs that matched the laser excitation wavelength at $532.2 \mathrm{~nm}( \pm 0.2 \mathrm{~nm})$ were utilized for the PPT heating. ${ }^{32}$ It is worth noting that under the ATR conditions with a $72^{\circ}$ inclined incident angle the plasmonic resonance wavelength for LSPR sensing transduction redshifted to $580 \mathrm{~nm}$ due to the prism coupling and the inclined angle of incidence (Figure 1e). ${ }^{30}$ The phase changes caused by a local variation of LSPR conditions were confined in a narrow wavelength region from 578 to $582 \mathrm{~nm}$. Moreover, after addition of a long-pass filter (LPF) with a cut-on wavelength at $552 \mathrm{~nm}$, the $532 \mathrm{~nm}$ photothermal excitation laser from the PPT unit did not influence the stability of the real-time LSPR sensing transduction. 

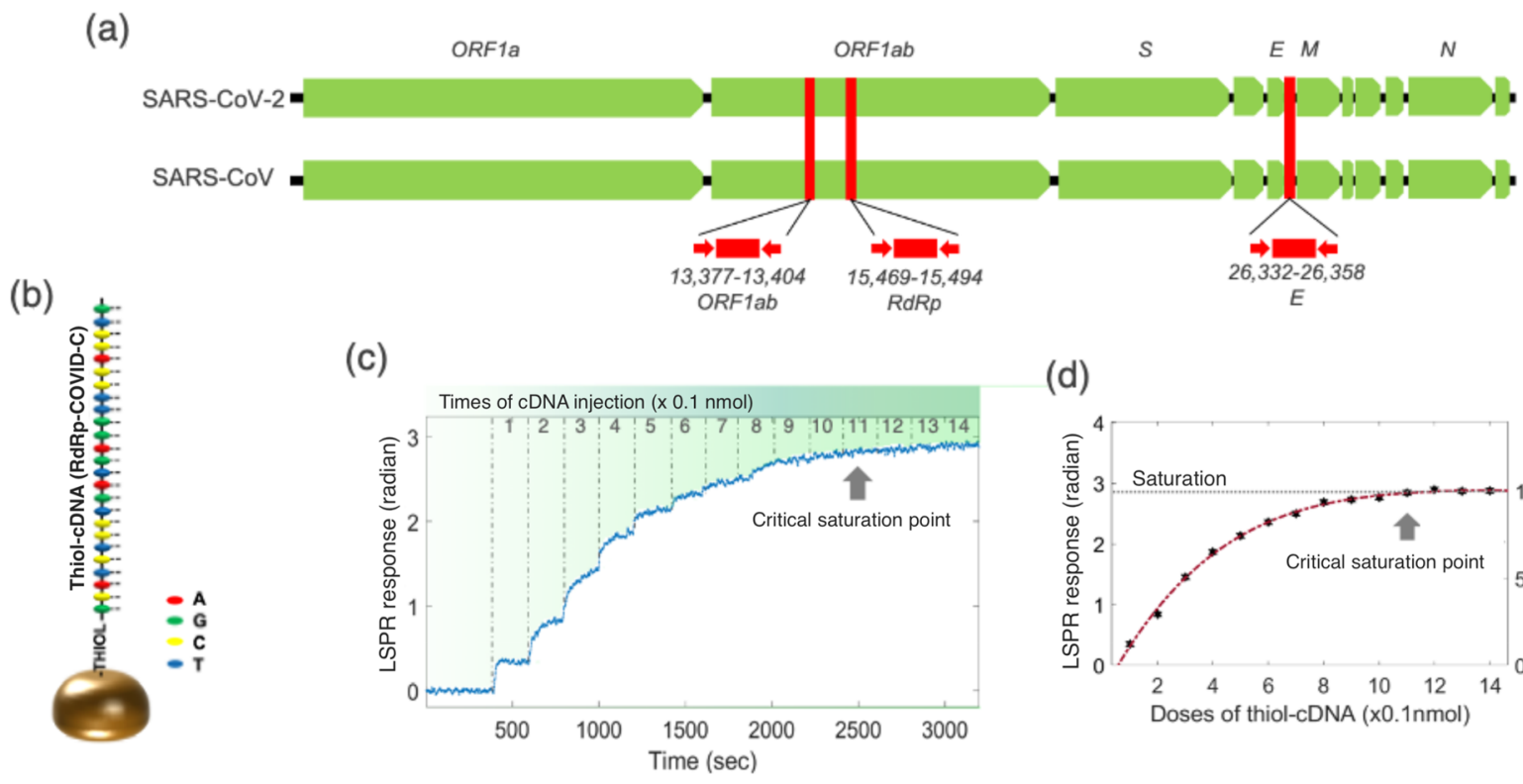

(d)

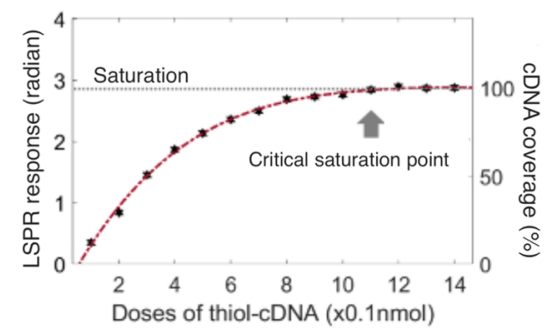

Figure 3. Selected viral sequences for SARS-CoV-2 detection. (a) Selected sequences and their relative positions used for SARS-CoV-2 and SARS-CoV detection. M: membrane protein gene; N: nucleocapsid protein gene; S: spike protein gene. The numbers below the sequences are genome positions according to GenBank, SARS-CoV-2 NC_045512. (b) Schematic illustration of AuNI functionalization based on the reaction with thiol-cDNA ligands. (c). Real-time monitoring of AuNI functionalization dynamics. Ten microliter solution containing 0.1 nmol of cDNA was injected in each step. (d) Calibrated surface functionalization efficiency to retrieve the optimal cDNA amount.

In the thermoplasmonic testing, the direct absorption of laser irradiation at $532 \mathrm{~nm}$ decayed nonradiatively by generating more hot electrons in AuNIs. ${ }^{33}$ The photoexcited highly energetic electrons quickly dissipated and released thermal energies to heat the ambient environments. Conversely, the PPT-induced temperature increase was also responsible for a refractive index variation of the surrounding environment, which can be in situ detected by the LSPR detection system as shown in Figure 2a. Specifically, the AuNI chip was exposed to laser excitation for $50 \mathrm{~s}$, as indicated by the shaded region. Then the laser was switched off to reattain the baseline. The generation and equilibrium of local photothermal heating were relatively fast. According to the laser switching tests as shown in Figure S2, the rapid heating process was completed within $1 \mathrm{~s}$ after turning on the laser excitation. Subsequently, the dynamic equilibrium process took another $11 \mathrm{~s}$ before finally entering the steady state. In our experiments, we calibrated the LSPR phase response under different ambient temperatures. The in situ temperature arising from the PPT effect was characterized based on the measurement of the thermal-induced refractive index variation in the vicinity of AuNIs. ${ }^{32,34}$ During the ambient temperature variation, the real-time LSPR phase responses and temperature values were recorded in parallel (Figure 2b), and the correlation was established as shown in Figure 2c. Based on this calibrated LSPR-temperature regression, the localized photothermal temperatures under different laser powers were retrieved as shown in Figure $2 \mathrm{~d}$.

To further evaluate the laser-induced PPT effect and the local temperature profile, we utilized the spectrometer to scan the heating area for mapping the LSPR phase responses and actual temperature distribution on the AuNI sensor chips. In the experimental setup as shown in Figure S3, the excitation laser with $32 \mathrm{~mW}$ power was applied to the optimal AuNI absorbers with a peak absorption at $532.2 \mathrm{~nm}( \pm 0.2 \mathrm{~nm})$. At each point of interest, we used the LSPR transducing unit to record two interferometric spectra: one reference without PPT heating and one spectrum with PPT heating. By scanning the laser spot and surrounding area with a $0.5 \mathrm{~mm}$ step interval, the spatial distribution of LSPR phase changes was retrieved as shown in Figure 2e. At each scanning pixel, the retrieved phase response was subsequently converted to the local temperature based on the calibration curve in Figure 2c. Therefore, the corresponding temperature distribution around the PPT heating was obtained and illustrated in Figure $2 \mathrm{f}$. The local temperature was significantly elevated from $21.47{ }^{\circ} \mathrm{C}$ (room temperature) to $41.08{ }^{\circ} \mathrm{C}$ at the center of the laser spot.

We shall now present the sensing results of the SARS-CoV-2 by the proposed dual-functional plasmonic biosensors. The full genome sequence data of the viruses, i.e., SARS-CoV-2 and SARS-CoV, have been retrieved from the GISAID platform. The selected oligonucleotides for specific SARS-CoV-2 detection and their relative positions were given in Figure 3a and Table S1. These viral oligonucleotides refer to sequences used in different countries for COVID-19 diagnosis, and some of them have been published in the latest research. ${ }^{8,9,35}$ The basic local alignment search tool (BLAST) was used to compare these viral sequences with the library of SARS-CoV-2 to confirm their representativeness and specificity. In the present case of COVID-19, SARS-CoV-2 isolates or samples from infected patients are challenging to obtain and handle. Thus, the corresponding DNA sequences were artificially synthesized for representative LSPR sensing demonstration of SARS-CoV-2 and SARS-CoV. According to the WHO guideline and local alignment searching results, two specific sequences from SARS-CoV-2 were selected, i.e., the RdRp and the ORF1ab as shown in Figure 3a. Validation and proof of selectivity were demonstrated by choosing the closely related nucleic acid sequence from RdRp of SARS-CoV. In addition, an oligonucleotide sequence from the coronaviral envelope protein gene (E) was also synthesized and tested to aid the virus identification. 
(a)
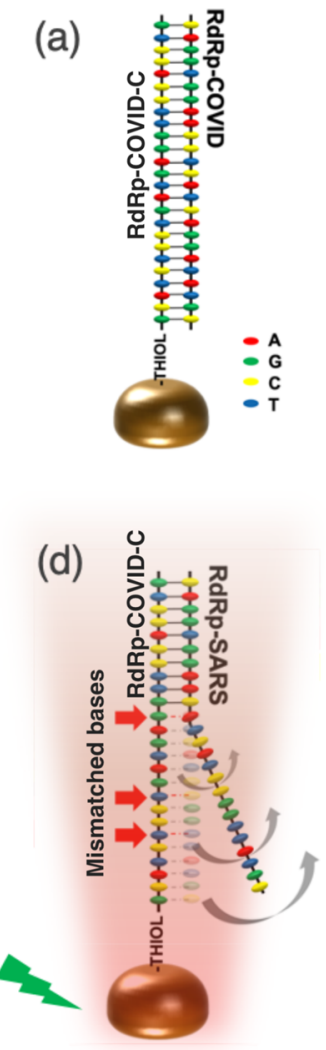

(b)

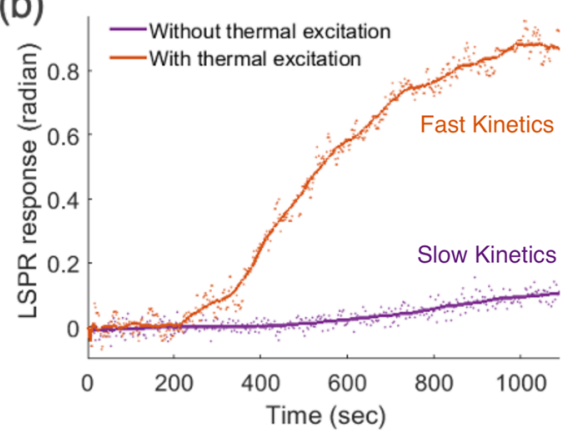

(e)

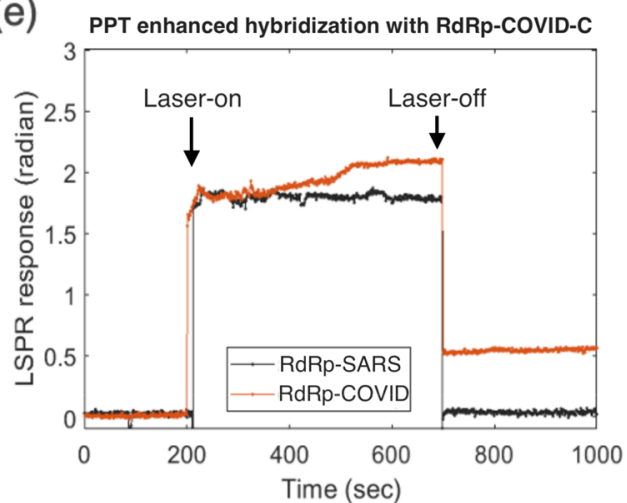

(c)

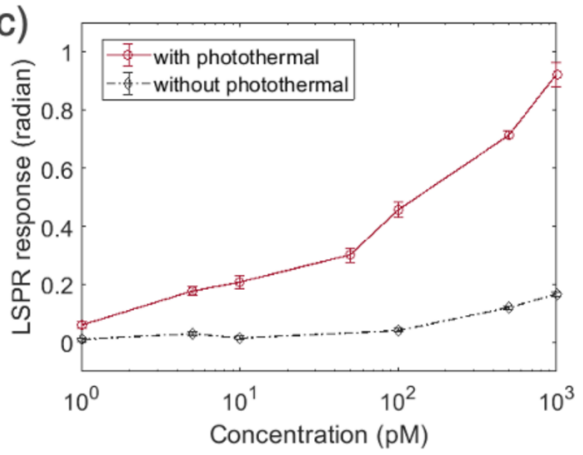

(f) PPT enhanced dissociation with RdRp-COVID-c

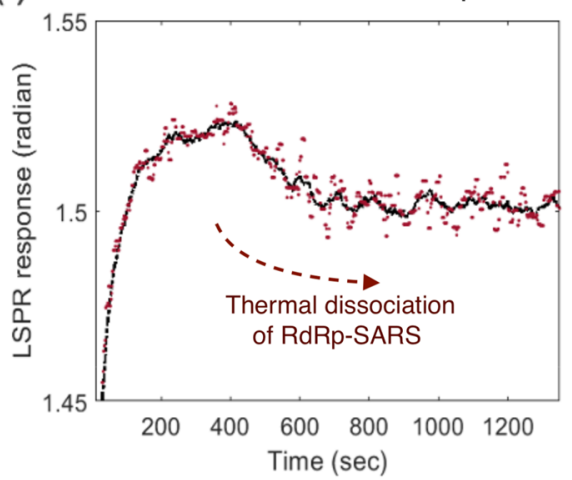

Figure 4. PPT enhancement in LSPR biosensing. (a) Schematic illustration of the hybridization of two complementary strands. (b) Realtime hybridization of RdRp-COVID and its cDNA sequence (RdRp-COVID-C) with or without the thermoplasmonic enhancement. (c) PPT enhancement on RdRp-COVID sequence detection at different concentrations. The error bars refer to the standard deviations of LSPR responses after reaching the steady conditions following the buffer flushing. (d) Schematic illustration of inhibited hybridization of two partially matched sequences. The red arrows indicated the mismatch bases of RdRp-SARS and functionalized cDNA of RdRp-COVID. (e) Discrimination of two similar sequences with PPT heat. The laser was applied at $200 \mathrm{~s}$ and switched off at $700 \mathrm{~s}$. (f) RdRp-SARS sequence dissociation from the immobilized RdRp-COVID-C sequence. The original phase responses (red dots) and the corresponding smoothed means (black curve) are shown.

Based on the synthetic oligonucleotide receptors with a thiol group (Table S2), the LSPR sensing chips were directly functionalized by forming the $\mathrm{Au}-\mathrm{S}$ bond between the thiolcDNA receptor and AuNIs as illustrated in Figure $3 \mathrm{~b}$. The surface functionalization process was initially optimized on its amount and concentration in order to achieve proper surface coverage and high sensitivity. During the real-time surface functionalization as shown in Figure 3c, step-by-step injections of $0.1 \mathrm{nmol}$ of thiol-cDNA of RdRp-COVID, (RdRp-COVIDC) caused continuous phase jumps due to the covalent binding between AuNIs and thiol-cDNA. After a total immobilization of $1 \mathrm{nmol}(10 \times 0.1 \mathrm{nmol})$ of RdRp-COVID-C as shown in Figure $3 \mathrm{c}, \mathrm{d}$, the LSPR response stopped growing and indicated the appropriate amount of cDNA receptors for AuNI functionalization. Hereafter, the solution containing $1 \mathrm{nmol}$ of thiol-cDNA was utilized to functionalize the AuNI microfluidic sensor chips for SARS-CoV-2 sequence detection (Figure S4). The proper surface functionalization that is sufficient to functionalize the entire AuNI sensing surface can increase the sensitivity and suppress the nonspecific binding events. In contrast, the AuNI sensor chip was oversaturated when functionalized with $10 \mathrm{nmol}$ of cDNA and insufficiently covered by using $0.1 \mathrm{nmol}$ of cDNA (Figure S5).

The surface-functionalized AuNI chips were subsequently installed in the LSPR systems for specific viral sequence detection (Figure 4a). The impacts of the localized thermoplasmonic heating on nucleic acids hybridization and LSPR detection were systematically studied. According to the temperature profile shown in Figure 2f, the excited PPT heat with approximately $41{ }^{\circ} \mathrm{C}$ nominal temperature was generated on the AuNI sensor. Before the injection of the RdRp sequence, nuclease-free water was flown across the microfluidic sensing chamber and the thermoplasmonic laser $(32 \mathrm{~mW})$ was turned on to establish a steady phase reference and baseline. According to the phase-sensing diagram in Figure $4 \mathrm{~b}$ and S6a, the LSPR response of the dual-functional AuNI biosensor started to increase when the RdRp-COVID genes were injected into the microfluidic chamber at about $200 \mathrm{~s}$ and attained the maximum phase value after about $800 \mathrm{~s}$ hybridization. The dual-functional AuNI sensing chip was further flushed with nuclease-free water to remove the nonspecific binding items and to check the final LSPR phase response. In the comparison with and without the PPT effect, the hybridization rate and the LSPR sensing response level were obviously suppressed when the PPT unit was shut down as shown in Figure $4 \mathrm{~b}$. It proved that the localized photothermal effect can significantly improve the hybridization kinetics of the RdRp-COVID and its cDNA. Thus, the response-slope of the photothermal enhanced LSPR was much steeper than that without the photothermal assistance. Due to the faster hybridization kinetics, the differential phase response levels were also elevated for the RdRp-COVID sequence at 
(a)

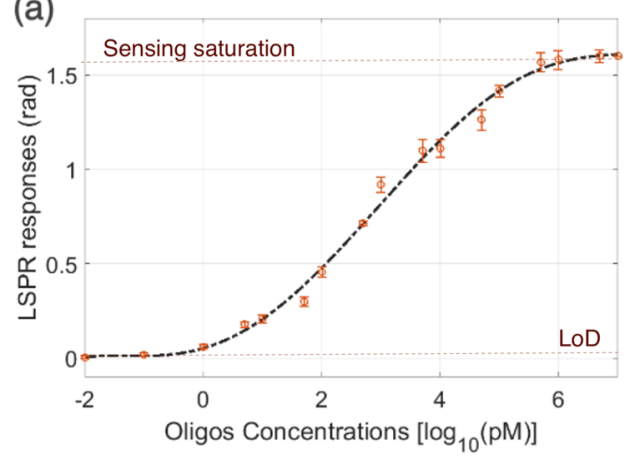

(c)

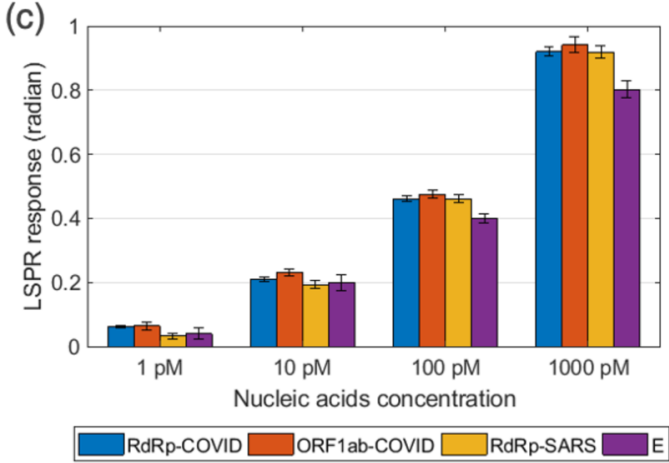

(b)

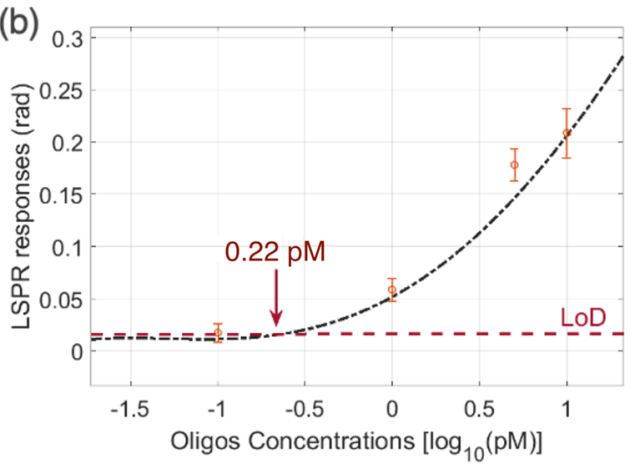

(d)

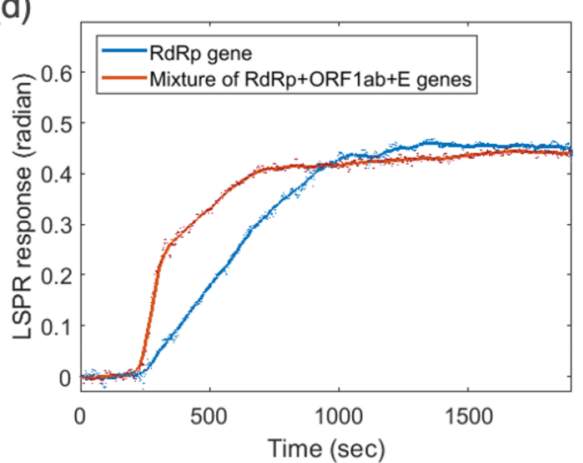

Figure 5. Evaluation of the dual-functional LSPR biosensor performance on detecting viral nucleic acids. (a) Plot of LSPR phase responses versus RdRp-COVID oligos concentrations using the PPT enhanced LSPR biosensor. (b) Zoom-in view of the low concentration range for LoD identification. (c) Concentrations of various viral oligos measured using the dual-functional LSPR biosensors. (d) Detection comparison of single analyte RdRp-COVID and mixture of multiple sequences. The error bars refer to the standard deviations of LSPR responses after reaching the steady conditions following the buffer flushing.

different concentrations as shown in Figure 4c and Figure S6. The PPT effect and its derived local heat can effectively promote the fast and sensitive detection of nucleic acids by improving the hybridization kinetics of fully matching strands.

More importantly, the PPT heating was capable of inhibiting the spurious binding of nonmatching sequences by elevating the local temperature at the vicinity of AuNIs. SARS-CoV and SARS-CoV-2 viruses are similar $\beta$-coronavirus, and their genetic similarities are high. The specific SARS-CoV-2 genetic target recommended by the WHO, i.e., the RdRp-COVID sequence as shown in Table $\mathrm{S} 1$, is very closely related to that of SARS-CoV. Specifically, in the selected gene sequences, only three fixed nucleotide bases were different between RdRpCOVID and RdRp-SARS. A real-time LSPR detection was conducted on the two closely related sequences. The LSPR sensor without the aid of photothermal unit reported a false positive response signal when detecting the RdRp-SARS sequence (Figure S7), which indicated that a similar but not fully complement sequence was also able to interact and partially hybridize with the cDNA receptors at room temperature. Although the hybridization kinetics of RdRpSARS sequence from SARS-CoV was clearly slower than that of SARS-CoV-2, the nonmatching spurious binding of any closely related sequence can affect the accurate virus detection and discrimination. Therefore, the local heat based on the proposed PPT effect was employed to improve the specificity of hybridization. At the elevated temperature of $41{ }^{\circ} \mathrm{C}$ as illustrated in Figure $4 \mathrm{~d}$, the standard free energy of hybridization was weaker due to the mismatched base-pairs. Thus, the similar but not fully matched sequences of SARS$\mathrm{CoV}$ can be distinguished. In detail, the calculated association rate constant $k_{\mathrm{a}}$ of RdRp-COVID with PPT heating enhancement was found to be $1.11 \times 10^{6} \mathrm{M}^{-1} \mathrm{~s}^{-1}$. A detailed discussion and calculations are given in Figure S6c. For a typical biological sensing system, $k_{\mathrm{a}}$ ranges between $10^{3}$ and $10^{7} \mathrm{M}^{-1} \mathrm{~s}^{-1}$ and a higher associate rate indicates a stronger binding affinity. ${ }^{36,37}$ In a comparison experiment including the PPT heat with $32 \mathrm{~mW}$ optical power, the $532 \mathrm{~nm}$ laser was applied onto the surface of the AuNI sensor from 200 to $700 \mathrm{~s}$ as shown in Figure 4e. The local PPT heat was generated immediately to make the LSPR phase jump to about $1.76 \mathrm{rad}$. After turning off the laser at $700 \mathrm{~s}$, the LSPR phase response of the mismatching RdRp-SARS gene was fully suppressed to the ground state of blank measurement (i.e., the responses from 0 to $200 \mathrm{~s}$ ) as shown by the black curve in Figure 4e. Since the RdRp-SARS sequences reported a weak response of $0.002 \mathrm{rad}$, we determined that its association rate constant was lower than $10^{3} \mathrm{M}^{-1} \mathrm{~s}^{-1}$ under the PPT heating. At the same time, the fully matching RdRp-COVID sequence from SARS-CoV-2, showed an apparent phase difference before and after the laser excitation (orange curve in Figure 4e). Thus, we believed that a similar but not fully matched sequence could be distinguished based on their different binding affinity and the PPT heating.

In another set of verification experiments, the RdRp-SARS genes were initially bound to the RdRp-COVID-C receptors at room temperature. Then the $532 \mathrm{~nm}$ laser $(32 \mathrm{~mW})$ was applied on the AuNI surface to stimulate the local thermoplasmonic effect. In the real-time LSPR sensorgram shown in Figure 4f, we observed the dissociation of the RdRpSARS genes from the RdRp-COVID-C receptors after the temperature rise. The calculated dissociation rate constant was 
$8.287 \times 10^{-3} \mathrm{~s}^{-1}$ as shown in Figure S8. The dissociation halflife $t_{1 / 2}$, which indicated the time to dissociate half of the hybridized sequences, was $83.3 \mathrm{~s}$. In contrast, the complementary sequence of RdRp-COVID showed a much lower dissociation rate constant at $3.5 \times 10^{-6} \mathrm{~s}^{-1}$ and a long dissociation half-life time of $1.97 \times 10^{5} \mathrm{~s}$. These results further verified that the thermoplasmonic effect can eliminate the nonmatching hybridization quickly and promote the selective detection of the target sequence, so as to achieve highly accurate nucleic acid detection and virus differentiation. Compared with the conventional plasmonic biosensing system, we demonstrated how this proposed dual-functional plasmonic sensing system can be the basis of a reliable and easy-toimplement thermoplasmonic biosensing technique: it can significantly reduce the false-positive-rate and enhance the reliability in genetic diagnosis.

To quantify the sensing performance, the dual-functional plasmonic detections of RdRp-COVID were further investigated over the concentration range from $0.01 \mathrm{pM}$ to $50 \mu \mathrm{M}$ as shown in Figure 5a. The AuNI sensing system started to attain the saturation condition when the concentration of the RdRp-COVID sequence reached $1 \mu \mathrm{M}$. In contrast, the low RdRp-COVID concentration, i.e., $0.1 \mathrm{pM}$, only resulted in a weak phase response by $2.90 \times 10^{-3}$ radian (Figure $5 \mathrm{~b}$ ), which was close to the system blank measurement of $2.92 \times 10^{-3}$ radian. Thus, as illustrated in the sensing calibration curve in Figure $5 b$, the dual-functional LSPR sensing system exhibited a limit of the range from $0.1 \mathrm{pM}$ to $1 \mu \mathrm{M}$ for detecting oligonucleotides, covering 7 orders of magnitude. The calibrated regression curve was further used to estimate the limit of detection (LoD), which is defined by IUPAC (International Union of Pure and Applied Chemistry) as the sum of the blank measures, i.e., $2.92 \times 10^{-3}$ radian with the nuclease-free water buffer and triple of its standard deviation (Figure S9). Thus, the LoD of the photothermal enhanced LSPR sensing system was found to be $\left(2.92 \times 10^{-3}\right)+3 \times$ $\left(3.12 \times 10^{-3}\right)=0.0123 \mathrm{rad}$ as shown by the dashed line in Figure $5 b$. Therefore, the detectable RdRp-COVID sequence concentration corresponding to the systematic LoD was about $0.22 \pm 0.08 \mathrm{pM}$ (Figure S9). A $200 \mu \mathrm{L}$ analyte solution at this LoD concentration contained about $2.26 \times 10^{7}$ copies of the RdRp-COVID sequence. The actual size of SARS-CoV-2 is about 29.9 kilobases in length, which is 1000 times longer than the RdRp-COVID sequence used in this study. Thus, based on the LSPR signal-target size relationship, the estimated LoD for detecting the entire RNA strands from SARS-CoV-2 could be approximately $2.26 \times 10^{4}$ copies. $^{38}$ A recent study reported the viral loads of SARS-CoV-2 from different respiratory trace samples including the throat/nasal swabs and the sputum. Based on these clinical specimens collected from 82 infected individuals, the overall viral load soon after onset was higher than $1 \times 10^{6}$ copies $/ \mathrm{mL}$. $^{39}$ This indicated that our proposed dual-functional LSPR system has the potential for direct analysis of SARS-CoV-2 sequences in respiratory samples.

In addition to the RdRp-COVID sequence, we also validated our dual-functional LSPR sensing system by performing the selective hybridization detection on several different genome sequences from both SARS-CoV-2 and SARS-CoV, i.e., the ORF1ab-COVID sequence and the E sequence from SARS$\mathrm{CoV}-2$, the RdRp-SARS sequence from SARS-CoV. The corresponding LSPR phase sensing responses with the in situ PPT enhancement are illustrated in Figure 5c. The complementary cDNA sequences, i.e., ORFlab-COVID-C, E-
$\mathrm{C}$, and RdRp-SARS-C, were functionalized onto the AuNI chips, respectively, for the detection of specific viral sequence. Since the physical length and molecular weight were roughly same, the hybridization of these target sequences reported a similar LSPR phase response (Figure 5c). As the concentration increased from $1 \mathrm{pM}$ to $1 \mathrm{nM}$, the mean LSPR response levels of each sequence also climbed in a proportional manner, which further proved the feasibility of this dual-functional LSPR sensing system for quantitative analysis of viral nucleic acids. Among them, the ORF1ab-COVID sequence produced the strongest responses due to its high molecular weight (8715.6 $\mathrm{g} / \mathrm{mol}$ ) and long length (28 bases), while the responses for $\mathrm{E}$ sequence were slightly lower.

In clinical diagnosis, the respiratory trace samples after viral lysis and RNA extraction may contain multiple nucleic acid sequences from the same viral source of SARS-CoV-2. Thus, detecting the accurate concentration of a specific sequence under the interference of multiple nonspecific sequences was beneficial to demonstrate its potential for real clinical applications. In experiments as shown in Figure $5 \mathrm{~d}$, the multisequence mixture containing RdRp-COVID sequences (100 pM), E sequences (100 pM), and ORF1ab-COVID sequences $(100 \mathrm{pM})$ was prepared to simulate an actual sample after virus lysis. The ORF1ab-COVID and E sequences in the mixture showed extremely low spurious binding with the immobilized RdRp-COVID-C receptors. Compared with the standard detection of $100 \mathrm{pM}$ RdRp-COVID as shown in Figure $5 \mathrm{~d}$, the calculated recovery rate based on the dualfunctional LSPR biosensors was found to be $96 \%$ in the mixture sample. This experimental result further demonstrated that the dual-functional LSPR system with the in situ PPT enhancement can perform accurate detection of the target sequence and facilitate the highly accurate SARS-CoV-2 detection.

\section{CONCLUSIONS}

Our developed dual-functional plasmonic system has successfully demonstrated a highly sensitive, fast, and reliable diagnostic capability for SARS-CoV-2 virus detection. This dual-functional plasmonic biosensing concept integrated the PPT effect and the LSPR sensing transduction on a single costeffective AuNI chip. By using two different angles of incidence, the plasmonic resonances of PPT and LSPR can be excited at two different wavelengths, which significantly enhanced the sensing stability, sensitivity, and reliability. With this configuration, the LSPR sensing unit attained a real-time and label-free detection of viral sequences including RdRp-COVID, ORFlab-COVID, and E genes from SARS-Cov-2. More importantly, the in situ PPT enhancement on the AuNI chips dramatically improved the hybridization kinetics and the specificity of nucleic acid detection. Similar sequences such as RdRp genes from SARS-CoV and SARS-CoV-2 can be accurately discriminated with the in situ PPT enhancement. Under the outbreak background of COVID-19, this proposed dual-functional LSPR biosensor can provide a reliable and easy-to-implement diagnosis platform to improve the diagnostic accuracy in clinical tests and relieve the pressure on PCR-based tests.

\section{MATERIALS AND METHODS}

Materials. All chemicals were purchased from commercial suppliers and used without further purification. Nuclease-free water was purchased from ThermoFisher and used as the buffer for 
oligonucleotide dilution and LSPR detection. All selected oligonucleotides, including the RdRp-COVID, RdRp-SARS, ORF1abCOVID, E sequence, and their thiol-cDNA receptors, including the RdRp-COVID-C, RdRp-SARS-C, and ORF1ab-COVID-C, E-C, were synthesized and provided by Microsynth (Balgach, Switzerland). All AuNI sensor chips and fluidic sensing chambers were cleaned using absolute ethanol followed by rinsing with Milli- $\mathrm{Q}$ water before testing.

Synthesis of Dual-Functional AuNI Chip. The AuNI sensor chips were synthesized based on the self-assembly process of thermal dewetted $\mathrm{Au}$ nanofilm. The original magnetron-sputtered $\mathrm{Au}$ nanofilms were optimized in a thickness range from 5.0 to $5.2 \mathrm{~nm}$. Then the Au nanofilm was thermally annealed at $550{ }^{\circ} \mathrm{C}$ for $3 \mathrm{~h}$. The AuNIs were self-assembled on the BK7 glass surface. The visible light absorption of each AuNI sensor chip was measured to retrieve the optimal plasmonic resonance condition.

Dual-Functional LSPR System. In our interferometric LSPR phase sensing system, a white light sensing beam was generated by an LED source and subsequently linearly polarized by a polarizer (P1). The thin birefringent crystal (BC) added sufficient retardation into the two orthogonal components of the linearly polarized light, i.e., the $s$ - and $p$-components, to create the spectral interferogram. The BK7 prism was able to couple the incident light into the AuNI-dielectric interface at an inclined nominal incident angle of $72^{\circ}$ and excited the local electromagnetic fields in the vicinity of the AuNIs by the Kretschmann configuration. The plasmonic resonance wavelength for LSPR sensing transduction was found to be $580 \mathrm{~nm}$. The interferometric spectra were screened by an aperture-iris (I1/I2, Thorlabs) with a hole diameter of $0.5 \mathrm{~mm}$ and finally recorded by the spectrometer (AvaSpec, Avantes). In addition to this plasmonic transducing unit, a high-power $532 \mathrm{~nm}$ laser diode (LD, $532 \mathrm{~nm}$ peak wavelength, DJ532-40 Thorlabs) was used for PPT heating by illuminating the AuNI chips in the normal incident angle. A longwavelength pass filter (LPF, $552 \mathrm{~nm}$ cut-on wavelength) was used to block the excitation signal before the spectrometer. The ambient temperature was measured and recorded with digital temperature sensors (SHTC1, Sensirion) for LSPR-temperature calibration.

Surface Functionalization with Thiol-cDNA. The AuNI surface functionalization was investigated based on the step-by-step injection of $0.1 \mathrm{nmol}$ thiol-cDNA. In the sensing chamber, $90 \mu \mathrm{L}$ of nucleasefree water was initially injected to build the phase reference baseline for $400 \mathrm{~s}$. Then, each time a $10 \mu \mathrm{L}$ solution which contained $0.1 \mathrm{nmol}$ thiol-cDNA, e.g., the RdRp-COVID-C sequence was injected into the sensor chamber in every $200 \mathrm{~s}$, until no further phase changes were recorded. Based on the optimal result, the solution containing $1 \mathrm{nmol}$ cDNA was utilized to functionalize the AuNI chips for the following SARS-CoV-2 sequences detection.

Detection of SARS-CoV-2 Viral Sequences. After the probe immobilization, the desired concentration of target DNA in nucleasefree water $(200 \mu \mathrm{L})$ was introduced into the AuNI microfluidic chamber for $800 \mathrm{~s}$, and the hybridization reaction was allowed under the PPT heat ( $32 \mathrm{~mW}$ optical power at $532 \mathrm{~nm}$ ). In the LSPR sensing path, an aperture-iris with a hole diameter of $0.5 \mathrm{~mm}$ was used to screen the sensing beam entering the spectrometer, which corresponded to the ATR light from the center of the PPT heat. Experiments on the mismatched nucleic acids and multisequence mixtures were also conducted based on the dual-functional LSPR biosensors as described above. A stringent buffer flushing with nuclease-free water was conducted after the hybridization. The whole testing process was real-time recorded by the spectrometer for plasmonic phase detection.

\section{ASSOCIATED CONTENT}

\section{s) Supporting Information}

The Supporting Information is available free of charge at https://pubs.acs.org/doi/10.1021/acsnano.0c02439.

Absorbance spectra of AuNIs; temperature profile of PPT heating; PPT heating system for characterizing the temperature distribution; selected target sequences from
SARS-CoV-2 and SARS-CoV; complementary thiolcDNA for LSPR functionalization; microfluidic detection system; comparison of AuNI surface functionalization; PPT effect on real-time LSPR detection; discrimination of two similar sequences without PPT heat; dissociation rate constant of RdRp-SARS; blank measurement for LoD (PDF)

\section{AUTHOR INFORMATION}

\section{Corresponding Author}

Jing Wang - Institute of Environmental Engineering, ETH Zürich, Zürich 8093, Switzerland; Laboratory for Advanced Analytical Technologies, Empa, Swiss Federal Laboratories for Materials Science and Technology, Dübendorf 8600, Switzerland; 이이. orcid.org/0000-0003-2078-137X; Email: jing.wang@ifu.baug.ethz.ch

\section{Authors}

Guangyu Qiu - Institute of Environmental Engineering, ETH Zürich, Zürich 8093, Switzerland; Laboratory for Advanced Analytical Technologies, Empa, Swiss Federal Laboratories for Materials Science and Technology, Dübendorf 8600, Switzerland

Zhibo Gai - Department of Clinical Pharmacology and Toxicology, University Hospital Zurich, University of Zürich, Zürich 8091, Switzerland; Experimental Center, Shandong University of Traditional Chinese Medicine, Jinan 250355, PR China

Yile Tao - Institute of Environmental Engineering, ETH Zürich, Zürich 8093, Switzerland; Laboratory for Advanced Analytical Technologies, Empa, Swiss Federal Laboratories for Materials Science and Technology, Dübendorf 8600, Switzerland

Jean Schmitt - Institute of Environmental Engineering, ETH Zürich, Zürich 8093, Switzerland; Laboratory for Advanced Analytical Technologies, Empa, Swiss Federal Laboratories for Materials Science and Technology, Dübendorf 8600, Switzerland

Gerd A. Kullak-Ublick - Department of Clinical Pharmacology and Toxicology, University Hospital Zurich, University of Zürich, Zürich 8091, Switzerland; Mechanistic Safety, CMO \& Patient Safety, Global Drug Development, Novartis Pharma, Basel 4002, Switzerland

Complete contact information is available at: https://pubs.acs.org/10.1021/acsnano.0c02439

\section{Author Contributions}

G.Q., Z.G., and J.W. conceived the research ideas. G.Q. constructed the dual-functional plasmonic system for SARSCoV-2 detection in J.W.'s group. Z.G., G.K.-U. and Y.T. contributed to the design and analysis of oligonucleotides. J.S. and G.Q. contributed to the thermoplasmonic measurement. G.Q. conducted the experiments and data analysis. G.Q. and J.W. wrote the manuscript. All authors have discussed the results and have given approval to the final version of the manuscripts.

\section{Notes}

The authors declare no competing financial interest.

\section{ACKNOWLEDGMENTS}

The authors acknowledge support from the FIRST Micro \& Nanoscience Center in ETH Zürich and the China Scholarship 
Council. We also thank Dr. Ying Du for providing the microfluidic chip.

\section{REFERENCES}

(1) Huang, C.; Wang, Y.; Li, X.; Ren, L.; Zhao, J.; Hu, Y.; Zhang, L.; Fan, G.; Xu, J.; Gu, X. Clinical Features of Patients Infected with 2019 Novel Coronavirus in Wuhan, China. Lancet 2020, 395, 497-506.

(2) Lu, R.; Zhao, X.; Li, J.; Niu, P.; Yang, B.; Wu, H.; Wang, W.; Song, H.; Huang, B.; Zhu, N. Genomic Characterisation and Epidemiology of 2019 Novel Coronavirus: Implications for Virus Origins and Receptor Binding. Lancet 2020, 395, 565-574.

(3) Rothe, C.; Schunk, M.; Sothmann, P.; Bretzel, G.; Froeschl, G.; Wallrauch, C.; Zimmer, T.; Thiel, V.; Janke, C.; Guggemos, W. Transmission of 2019-nCoV Infection from an Asymptomatic Contact in Germany. N. Engl. J. Med. 2020, 382, 970.

(4) Stoecklin, S. B.; Rolland, P.; Silue, Y.; Mailles, A.; Campese, C.; Simondon, A.; Mechain, M.; Meurice, L.; Nguyen, M.; Bassi, C. First Cases of Coronavirus Disease 2019 (COVID-19) in France: Surveillance, Investigations and Control Measures, January 2020. Eurosurveillance 2020, 25, 2000094.

(5) Wang, L. F.; Anderson, D. E. Viruses in Bats and Potential Spillover to Animals and Humans. Curr. Opin. Virol. 2019, 34, 79-89.

(6) Ksiazek, T. G.; Erdman, D.; Goldsmith, C. S.; Zaki, S. R.; Peret, T.; Emery, S.; Tong, S. X.; Urbani, C.; Comer, J. A.; Lim, W.; Rollin, P. E.; Dowell, S. F.; Ling, A. E.; Humphrey, C. D.; Shieh, W. J.; Guarner, J.; Paddock, C. D.; Rota, P.; Fields, B.; DeRisi, J.; et al. A Novel Coronavirus Associated with Severe Acute Respiratory Syndrome. N. Engl. J. Med. 2003, 348, 1953-1966.

(7) Mahase, E. Coronavirus: COVID-19 Has Killed More People Than SARS and MERS Combined, Despite Lower Case Fatality Rate. Br. Med. J. 2020, M641.

(8) Corman, V. M.; Landt, O.; Kaiser, M.; Molenkamp, R.; Meijer, A.; Chu, D. K.; Bleicker, T.; Brünink, S.; Schneider, J.; Schmidt, M. L. Detection of 2019 Novel Coronavirus (2019-nCoV) by Real-Time RT-PCR. Eurosurveillance 2020, 25.

(9) World Health Organization. Laboratory Testing for Coronavirus Disease 2019 (Covid-19) in Suspected Human Cases: Interim Guidance, 2 March 2020; WHO/COVID-19/laboratory/2020.4; World Health Organization: Geneva, 2020; https://www.who.int/emergencies/ diseases/novel-coronavirus-2019/technical-guidance/laboratoryguidance (accessed March 2, 2020).

(10) Xie, X.; Zhong, Z.; Zhao, W.; Zheng, C.; Wang, F.; Liu, J., Chest CT for Typical 2019- nCoV Pneumonia: Relationship to Negative RT-PCR Testing. Radiology 2020, 200343.

(11) Zhang, Q.; Zhao, Q., Inactivating Porcine Coronavirus before Nucleic Acid Isolation with the Temperature Higher Than $56{ }^{\circ} \mathrm{C}$ Damages Its Genome Integrity Seriously. BioRxiv, 2020; DOI: 10.1101/2020.02.20.958785 (accessed March 2, 2020).

(12) Ye, Z.; Zhang, Y.; Wang, Y.; Huang, Z.; Song, B. Chest CT Manifestations of New Coronavirus Disease 2019 (COVID-19): A Pictorial Review. Eur. Radiol. 2020, 1-9.

(13) Soler, M.; Huertas, C. S.; Lechuga, L. M. Label-Free Plasmonic Biosensors for Point-of-Care Diagnostics: A Review. Expert Rev. Mol. Diagn. 2019, 19, 71-81.

(14) Masson, J. F. Surface Plasmon Resonance Clinical Biosensors for Medical Diagnostics. ACS Sens 2017, 2, 16-30.

(15) Haes, A. J.; Chang, L.; Klein, W. L.; Van Duyne, R. P. Detection of a Biomarker for Alzheimer's Disease from Synthetic and Clinical Samples Using a Nanoscale Optical Biosensor. J. Am. Chem. Soc. 2005, 127, 2264-2271.

(16) Willets, K. A.; Van Duyne, R. P. Localized Surface Plasmon Resonance Spectroscopy and Sensing. Annu. Rev. Phys. Chem. 2007, 58, 267-297.

(17) Anker, J. N.; Hall, W. P.; Lyandres, O.; Shah, N. C.; Zhao, J.; Van Duyne, R. P. Biosensing with Plasmonic Nanosensors. Nat. Mater. 2008, 7, 442-453.

(18) Qiu, G. Y.; Ng, S. P.; Wu, C. M. L. Bimetallic Au-Ag Alloy Nanoislands for Highly Sensitive Localized Surface Plasmon Resonance Biosensing. Sens. Actuators, B 2018, 265, 459-467.
(19) Qiu, G.; Thakur, A.; Xu, C.; Ng, S. P.; Lee, Y.; Wu, C. M. L. Detection of Glioma-Derived Exosomes with the Biotinylated Antibody-Functionalized Titanium Nitride Plasmonic Biosensor. Adv. Funct. Mater. 2019, 29, 1806761.

(20) Wrapp, D.; Wang, N.; Corbett, K. S.; Goldsmith, J. A.; Hsieh, C.-L.; Abiona, O.; Graham, B. S.; McLellan, J. S. Cryo-EM Structure of the 2019-nCoV Spike in the Prefusion Conformation. Science 2020, 367, 1260-1263.

(21) Zhang, D. Y.; Chen, S. X.; Yin, P. Optimizing the Specificity of Nucleic Acid Hybridization. Nat. Chem. 2012, 4, 208.

(22) Stehr, J.; Hrelescu, C.; Sperling, R. A.; Raschke, G.; Wunderlich, M.; Nichtl, A.; Heindl, D.; Kürzinger, K.; Parak, W. J.; Klar, T. A. Gold Nanostoves for Microsecond DNA Melting Analysis. Nano Lett. 2008, 8, 619-623.

(23) Zhang, J. X.; Fang, J. Z.; Duan, W.; Wu, L. R.; Zhang, A. W.; Dalchau, N.; Yordanov, B.; Petersen, R.; Phillips, A.; Zhang, D. Y. Predicting DNA Hybridization Kinetics from Sequence. Nat. Chem. 2018, 10, 91-98.

(24) Harris, N. C.; Kiang, C.-H. Defects Can Increase the Melting Temperature of DNA- Nanoparticle Assemblies. J. Phys. Chem. B 2006, 110, 16393-16396.

(25) Jauffred, L.; Samadi, A.; Klingberg, H.; Bendix, P. M.; Oddershede, L. B. Plasmonic Heating of Nanostructures. Chem. Rev. 2019, 119, 8087-8130.

(26) Lee, J. H.; Cheglakov, Z.; Yi, J.; Cronin, T. M.; Gibson, K. J.; Tian, B. Z.; Weizmann, Y. Plasmonic Photothermal Gold Bipyramid Nanoreactors for Ultrafast Real-Time Bioassays. J. Am. Chem. Soc. 2017, 139, 8054-8057.

(27) Son, J. H.; Cho, B.; Hong, S.; Lee, S. H.; Hoxha, O.; Haack, A. J.; Lee, L. P. Ultrafast Photonic PCR. Light: Sci. Appl. 2015, 4, No. e280.

(28) Kim, M.; Lee, J. H.; Nam, J. M. Plasmonic Photothermal Nanoparticles for Biomedical Applications. Adv. Sci. 2019, 6, 1900471.

(29) Wang, S.; Huang, P.; Nie, L.; Xing, R.; Liu, D.; Wang, Z.; Lin, J.; Chen, S.; Niu, G.; Lu, G. Single Continuous Wave Laser Induced Photodynamic/Plasmonic Photothermal Therapy Using Photosensitizer-Functionalized Gold Nanostars. Adv. Mater. 2013, 25, 30553061.

(30) Qiu, G. Y.; Ng, S. P.; Wu, C. M. L. Differential Phase-Detecting Localized Surface Plasmon Resonance Sensor with Self-Assembly Gold Nano-Islands. Opt. Lett. 2015, 40, 1924-1927.

(31) Smolyaninov, A.; El Amili, A.; Vallini, F.; Pappert, S.; Fainman, Y. Programmable Plasmonic Phase Modulation of Free-Space Wavefronts at Gigahertz Rates. Nat. Photonics 2019, 13, 431-435.

(32) Chen, Z. X.; Shan, X. N.; Guan, Y.; Wang, S. P.; Zhu, J. J.; Tao, N. J. Imaging Local Heating and Thermal Diffusion of Nanomaterials with Plasmonic Thermal Microscopy. ACS Nano 2015, 9, 1157411581.

(33) Baffou, G.; Quidant, R.; García de Abajo, F. J. Nanoscale Control of Optical Heating in Complex Plasmonic Systems. ACS Nano 2010, 4, 709-716.

(34) Baffou, G.; Bon, P.; Savatier, J.; Polleux, J.; Zhu, M.; Merlin, M.; Rigneault, H.; Monneret, S. Thermal Imaging of Nanostructures by Quantitative Optical Phase Analysis. ACS Nano 2012, 6, 2452-2458.

(35) Zhu, N.; Zhang, D. Y.; Wang, W. L.; Li, X. W.; Yang, B.; Song, J. D.; Zhao, X.; Huang, B. Y.; Shi, W. F.; Lu, R. J.; Niu, P. H.; Zhan, F. X.; Ma, X. J.; Wang, D. Y.; Xu, W. B.; Wu, G. Z.; Gao, G. G. F.; Tan, W. J. A Novel Coronavirus from Patients with Pneumonia in China, 2019. N. Engl. J. Med. 2020, 382, 727-733.

(36) Xu, S. C.; Zhan, J.; Man, B. Y.; Jiang, S. Z.; Yue, W. W.; Gao, S. B.; Guo, C. G.; Liu, H. P.; Li, Z. H.; Wang, J. H.; Zhou, Y. Q. RealTime Reliable Determination of Binding Kinetics of DNA Hybridization Using a Multi-Channel Graphene Biosensor. Nat. Commun. 2017, DOI: $10.1038 /$ ncomms 14902 .

(37) Schreiber, G.; Haran, G.; Zhou, H. X. Fundamental Aspects of Protein-Protein Association Kinetics. Chem. Rev. 2009, 109, 839-860.

(38) Qiu, G.; Yue, Y.; Tang, J.; Zhao, Y.-B.; Wang, J. Total Bioaerosols Detection by a Succinimidyl-Ester-Functionalized Plas- 
monic Biosensor to Reveal Different Characteristics at Three

Locations in Switzerland. Environ. Sci. Technol. 2020, 54, 1353-1362.

(39) Pan, Y.; Zhang, D.; Yang, P.; Poon, L. L.; Wang, Q. Viral Load

of SARS-CoV-2 in Clinical Samples. Lancet Infect. Dis. 2020, 20, 411. 